\title{
A Knowledge Service Framework based on Ontology
}

\author{
Si Chen ${ }^{a}$, De Zhang ${ }^{b}$, Guanghong Liu $^{c}$ and Wenhua Shen ${ }^{d}$ \\ ${ }^{1}$ Information Science Academy, China Electronics Technology Group Corporation, Beijing 100081, \\ China. \\ acs_cetc@163.com, b zddy7625 @sohu.com, c Ighonguan @aliyun.com, \\ d shenwenhua@sina.com
}

Keywords: Knowledge service, semantic space model, domain ontology, knowledge-based system.

\begin{abstract}
In order to improve the utilization ratio of knowledge and make the knowledge rise in value, a framework of knowledge service for design activities is proposed. Firstly, a product design knowledge representation model is proposed according to the characteristics of design knowledge, and then a knowledge service model based on semantic space is given. Then a method of knowledge service matching is proposed based on the knowledge requirements analysis. Finally, these approaches are used in a knowledge service system for military-vehicle design validating its effectiveness and feasibility.
\end{abstract}

\section{Introduction}

The process of product-design involved a lot of knowledge which is in varieties of style, such as standard specification, design manual, formula, and design prototype and so on[1], as the distribution and the diversity expression of the product-design knowledge, enterprises lack a unified expression pattern of design resources, and utilize the design knowledge inefficiently. Recent years, many researches have been done which mainly focus on two points: one is to study how to acquire and represent product-design knowledge, and the other is to discuss how to get design knowledge what the user really need swiftly and accurately. In the process of product design, designers get knowledge often through two ways that are knowledge retrieval and knowledge pushing. But the traditional method of knowledge retrieval or knowledge pushing is based on keyword and ignores the semantic relationship between knowledge. Keywords could represent only fragmented meanings of the content, and the content represented through keywords did not always meet the user's requirements. On the other hand, in the product design process, just using the product design knowledge itself as a reference can't meet the designers' requirements. They usually hope a complete solution of a design problem can be given through knowledge reasoning and data mining.

Therefore, there need a new approach to get knowledge swiftly and accurately and make the knowledge rise in value greatly in product-design process. So this paper proposes a framework for knowledge service oriented product design activities. The details will be illustrated in next sections. Finally an example of application will be showed to demonstrate that this approach is effective and feasible.

\section{A Framework of Knowledge Service}

\subsection{Definition of Knowledge Service.}

The section headings are in boldface capital and lowercase letters. Second level headings are typed as part of the succeeding paragraph (like the subsection heading of this paragraph). All manuscripts must be in English, also the table and figure texts, otherwise we cannot publish your paper. Please keep a second copy of your manuscript in your office. When receiving the paper, we assume that the corresponding authors grant us the copyright to use the paper for the book or journal in question. When receiving the paper, we assume that the corresponding authors grant us the copyright to use the paper 
for the book or journal in question. When receiving the paper, we assume that the corresponding authors grant us the copyright to use.

With the development of product design knowledge management, in recent years a lot of researches on product design knowledge service have been done by scholars at home and abroad. But there is no general definition of product design knowledge service. Yang Tao [2] proposes that knowledge service system is a kind of intelligent design assistant, and in the design process provide active knowledge service for designers through capturing and analyzing designers' action. Dong Ying [3] points out that knowledge service is a process of transforming knowledge asset to knowledge product and service, and selling the service through the internet. Gregoris M[4] thinks that knowledge service is a commercial method of mining knowledge assets, and contributes to improving enterprise competitive advantage and expanding the market share.

The knowledge service this paper studied is orientated on product design activities. This paper integrates all kinds of viewpoints and our own research object's characteristics, and then a definition of knowledge service orientated design activities is given.

Definition 1: the knowledge service orientated product design activities is a process of precisely and actively providing knowledge to the designer in real time according to knowledge requirement of design activities, and making the knowledge maximally increased in value in product design process.

The knowledge service emphasizes four characteristics that are "in real time", "accurately", "actively", "value addable", and compared to the knowledge retrieval or push of traditional knowledge management, knowledge service pay more attention to how to embody the added value of knowledge. In the process of traditional knowledge management, knowledge is used just to be read or referenced, and the value of knowledge doesn't get further mining and processing. However, in the process of knowledge service, on the basis of accurately acquiring knowledge which is the designer really demands, the value of knowledge can be increased maximally.

\subsection{The Framework of Knowledge Service.}

In order to using the existing knowledge effectively, a framework of knowledge service oriented product design activities is proposed which includes four layers: target layer, method layer, technology layer and resources layer. The framework is shown as Fig.1.

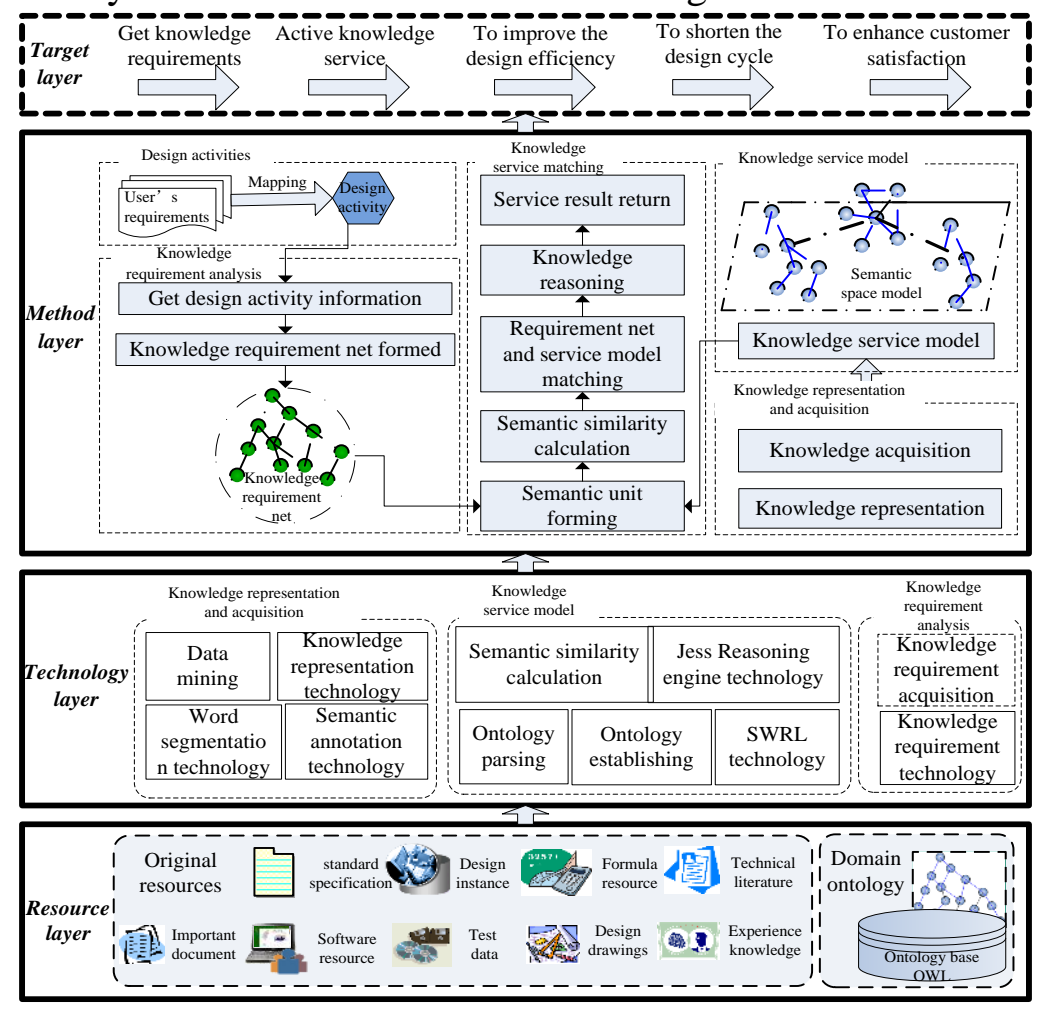

Fig. 1 The Framework of Knowledge Service

1) Target layer: The purpose of knowledge service oriented product design activities is to improve the efficiency of product design and shorten delivery cycle and improve customer satisfaction. 
2) Method layer: Method layer describes the overall idea for this study. Firstly, the knowledge should be acquired from the original resources and then stored in knowledge base based on the knowledge representation model. In order to meet the demand of knowledge service, the original knowledge base knowledge should be organized by knowledge service organization model. This paper proposes semantic space to express the logical relationship between knowledge. A perfect knowledge service organization model can be formed through the mergence and consistency test of semantic space. And then the knowledge requirement which is from a product design activity in process or an independent product design activity should be acquired. There are all kinds of knowledge requirements from a design activity. This paper proposes knowledge requirement net to modeling knowledge requirements. Then the knowledge requirement net will match the service organization model which is based on semantic space. In the matching process, knowledge is acquired and the value of knowledge is increased. At last, the result of service knowledge will be returned to requester.

3) Technology layer: Some technologies which will be used in method layer are described in technology layer, such as technology of segmentation, semantic annotation, semantic similarity computation, and reasoning engine and so on.

4) Resource layer: The underlying resource pool of an enterprise which includes a lot of existing knowledge resources, such as important reports, standards, intelligence information, technical documents, experience knowledge, design instances, design models, expert resources, software resources, formulas, and domain ontology resources. Because not only the knowledge representation and acquisition method this paper proposed is based on ontology but also the formation of semantic space, so a perfect domain ontology is necessary.

\section{Knowledge Representation and Service Model Establishment}

\subsection{Multi-dimensional Domain Ontology Modeling.}

Product design knowledge contains rich semantic information. In order to express and understand this semantic information, product design knowledge should be represented and organized by domain ontology. In order to manage the product-design knowledge efficiently, establishing the product and design process ontology is necessary. In computer science, ontology is usually understood as a "formal, explicit specification of a shared conceptualization" [6]. Actually ontology is a formal model that explicitly represents the consensual knowledge of a domain. The domain entities are modeled by classes and relations. Domain ontology is a set of terms and relationship of a certain field. The basic elements of ontology include classes, relationships, rules, axioms and instances. Ontology as an important tool for knowledge and concept representation, provides the perfect means to describe domain objects, and additionally, to describe the interrelationships among them as well as the rules.

A method of multi-dimensional domain ontology modeling is used in this paper. The domain ontology is established from four dimensions: design object dimension, design organization dimension, design process dimension and knowledge object dimension.

\subsection{Knowledge Unit Representation.}

Knowledge representation is the foundation of knowledge management and knowledge service. In order to facilitate the implementing of knowledge acquisition and knowledge service, a knowledge representation method based on knowledge unit is proposed.

Definition2 knowledge unit: knowledge unit is an independent and smallest data unit which can completely describe product design knowledge such as design experience, design rules and design instance. Knowledge unit $\left(K_{u}\right)$ is represented as shown following:

$$
K_{u}=\left[\begin{array}{lll}
T_{1} & T_{2} \ldots & T_{n} \\
C_{1} & C_{2} \ldots & C_{n}
\end{array}\right]
$$

The Ti is the subject heading of the knowledge unit; The $\mathrm{Ci}$ is a string that describes detail contents of $\mathrm{Ti}, \mathrm{n}$ is the number of theme of the knowledge unit. In addition, if there are so many knowledge units, 
they can be represented as $\mathrm{Ku} 1, \mathrm{Ku} 2, \mathrm{Ku} 3, \mathrm{Kum}$. The theme of knowledge unit should as far as possible use the concepts of the domain ontology.

\subsection{The Framework of Knowledge Service Model.}

The knowledge units are messy in the knowledge base, so a knowledge service organized modeling is needed to organize these knowledge units. The framework of knowledge service organized modeling this paper proposed is shown as Fig.2.This model contains three layers: knowledge unit layer, knowledge module layer and semantic space layer.

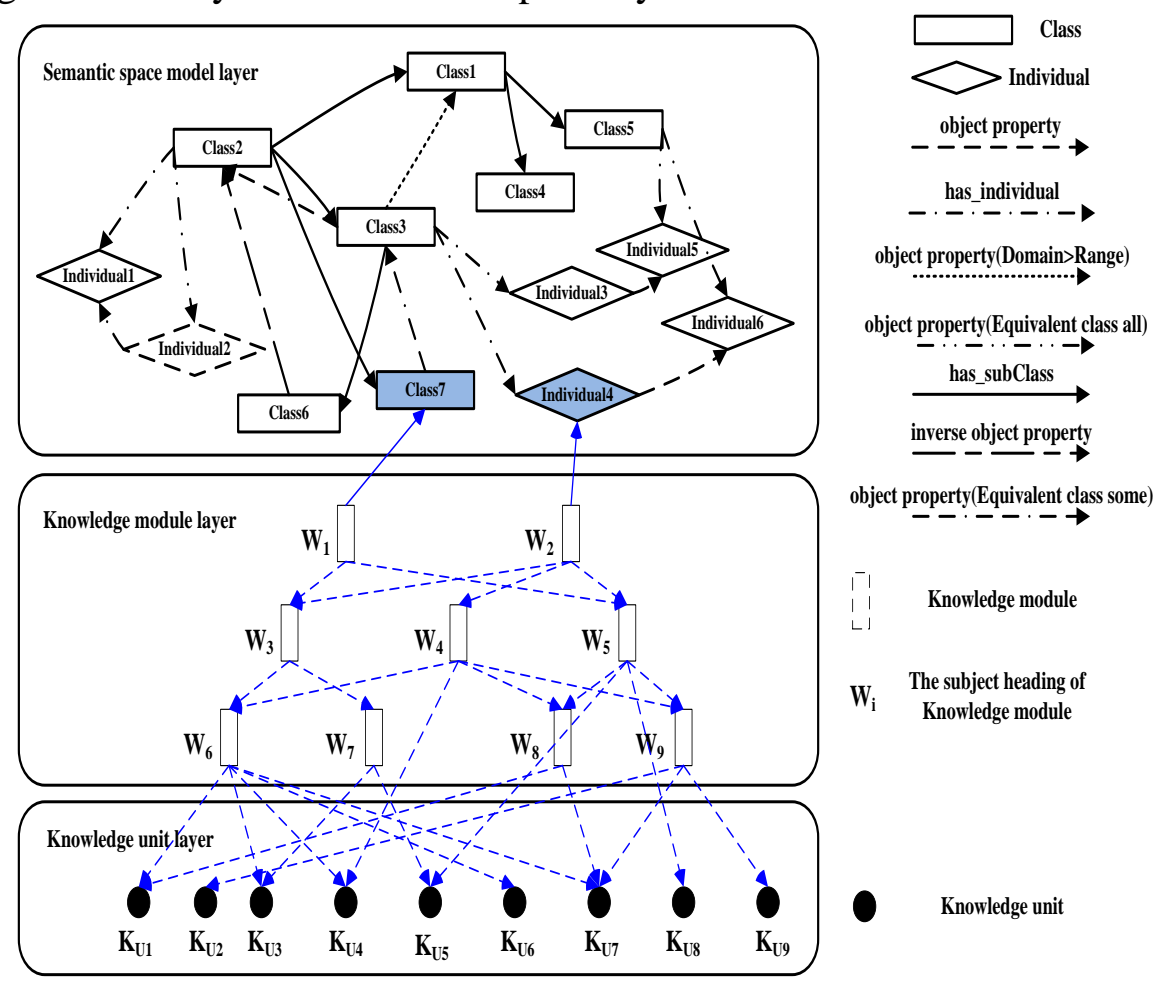

Fig. 2 Knowledge Service Model

To establish knowledge service organization model should follow two steps: 1) generating knowledge module according to the similarity between the themes of knowledge unit. 2) Forming semantic space through the ontology extension based on knowledge module.

\subsubsection{Knowledge module}

Knowledge module is a combination of knowledge units which have the similar subject heading. Its condition of combination is as following:

Condition 1: there is a concept $\mathrm{W}$ in domain ontology;

Condition2: Any knowledge unit $\mathrm{K}_{\mathrm{ui}}$ has a subject heading $\mathrm{T}_{\mathrm{ij}}$ (content is the $\mathrm{C}_{\mathrm{ij}}$ ) in $\left\{\mathrm{Ku}_{1}, \mathrm{Ku}_{2} \cdots \mathrm{Ku}_{\mathrm{m}}\right\}$;

Condition3: $\mathrm{W}, \mathrm{T}_{\mathrm{ij}}$ and $\mathrm{C}_{\mathrm{ij}}$ in condition 1 and 2 meet the conditions $\left(\mathrm{T}_{\mathrm{ij}}=\mathrm{W}\right) \cup\left(\mathrm{T}_{\mathrm{ij}} \propto \mathrm{W}\right) \cup\left(\mathrm{C}_{\mathrm{ij}}=\mathrm{W}\right) \cup\left(\exists \mathrm{w}\left(\mathrm{w} \in \mathrm{C}_{\mathrm{ij}}\right) \cap(\mathrm{w} \propto \mathrm{W})\right)$

The $\exists \mathrm{w}\left(\mathrm{w} \in \mathrm{C}_{\mathrm{ij}}\right) \cap(\mathrm{w} \propto \mathrm{W})$ in condition3 represents that there exists a string w whose semantic similarity is close to $\mathrm{W}$.

Let's hypothesize that there is a knowledge module $K_{M}$ which is a combination of $\left\{\mathrm{Ku}_{1}\right.$, $\left.\mathrm{Ku}_{2} \ldots \mathrm{Ku}_{\mathrm{m}}\right\}$. There is a candidate subject heading set $\left\{\mathrm{W}_{1}, \mathrm{~W}_{2} \ldots \mathrm{W}_{\mathrm{n}}\right\}$. If the $\mathrm{W}_{\mathrm{i}}$ satisfy the following conditions:

$$
\mathrm{d}\left(\mathrm{W}_{i}, \text { root }\right)=\max \left\{\mathrm{d}\left(\mathrm{W}_{1}, \text { root }\right), \mathrm{d}\left(\mathrm{W}_{2}, \text { root }\right), \ldots ., \mathrm{d}\left(\mathrm{W}_{i}, \text { root }\right)\right\}
$$

$\mathrm{W}_{\mathrm{i}}$ is choosed as a subject heading of $\mathrm{K}_{\mathrm{M}}$. The $\mathrm{d}\left(\mathrm{W}_{j}\right.$, root $)$ in condition represents the distance between the concept $\mathrm{W}_{\mathrm{j}}$ and root concept in the domain ontology.

Knowledge modules also can be combined until the semantic distance between their subject headings increases to a certain value. Finally if the count of knowledge module is $\mathrm{m}$, a vector space 
$K=\left\{k_{1}, k_{2}, \ldots, k_{m}\right\}$ of $\mathrm{m}$ dimension will be established. The vector space is called final expression vector knowledge module.

\subsubsection{Semantic Space Model}

The semantic relationship between the subjects heading is not tight, so a semantic space model which based on ontology is needed to organize the knowledge modules. Semantic space model can be generated by the following steps: 1) ontology parser; 2) semantic matching; 3) semantic space model generation.

\subsubsection{Ontology parsing}

The domain ontology should be parsed to a set of ontology elements vector before matching with the vector of knowledge module. The Class vector, individual vector, object property vector, dada property vector and description vector willed be formed after ontology parsing. The original ontology parser vector can be represented as:

$$
\begin{aligned}
& \text { OWLClass }_{\text {ini }}=\left\{C_{1}, C_{2}, \ldots ., C_{n}\right\} \\
& \text { OWLIndividuals }_{i n i}=\left\{I_{1}, I_{2}, \ldots, I_{o}\right\} \\
& \text { OWLObject } \operatorname{Pr} \text { operty }_{i n i}=\left\{O_{1}, O_{2}, \ldots ., O_{p}\right\} \\
& \text { OWLData Pr operty }{ }_{\text {ini }}=\left\{D_{1}, D_{2}, \ldots ., D_{q}\right\} \\
& \text { OWLDescription }_{i n i}=\left\{\text { Des }_{1}, \text { Des }_{2}, \ldots ., \text { Des }_{r}\right\}
\end{aligned}
$$

OWLClass $_{\text {ini }}$ represents the original vector of class. OWLIndividuals $s_{i n i}$ represents the original vector of individual. OWLObject Pr operty $_{i n i}$ represents the original vector of object property. OWLData Property ${ }_{i n i}$ represents the original vector of data property. $O W L D_{\text {Description }}{ }_{i n i}$ represents the original vector of description.

\subsubsection{Semantic matching}

Semantic matching is a process of the vector of knowledge module matching with the five vectors of ontology elements separately. The similarity calculation arithmetic will be introduced in more detail.

The elements of vector of knowledge module $K=\left\{k_{1}, k_{2}, \ldots, k_{m}\right\}$ and vector of ontology class OWLClass $_{\text {ini }}=\left\{c_{1}, c_{2}, \ldots, c_{n}\right\}$ match with each in turn. Actually, it is the similarity calculation of two strings. In this paper, a similarity calculation similarity is proposed based on Levenshtein Distance and LCS (Longest Common Subsequences) arithmetic.

The formula of Levenshtein Distance is given in [7]:

$$
\operatorname{Sim}_{L D}(K, C)=1-N E D(K, C)
$$

The $N E D(K, C)$ is the distance of string $\mathrm{K}$ and $\mathrm{C}$.

The formula LCS is given in [8]:

$$
\operatorname{Sim}_{L C S}(K, C)=\frac{2 \times \text { LCSLength }}{|K|+|C|}
$$

The $|K||C|$ is the length of string $\mathrm{K}$ and $\mathrm{C}$, LCSLength is the length of the longest common subsequences.

This paper proposes a formula of semantic similarity calculation:

$$
\operatorname{Sim}(K, C)=\alpha \times \operatorname{Sim}_{L D}(K, C)+\beta \times \operatorname{Sim}_{L C S}(K, C), \alpha+\beta=1
$$

A matrix of semantic similarity which is $n \times m$ will be formed after the elements of $K=\left\{k_{1}, k_{2}, \ldots, k_{m}\right\}$ and $O W L C l a s s_{i n i}=\left\{c_{1}, c_{2}, \ldots, c_{n}\right\}$ matching with each other in turn.

$$
S=\left[\begin{array}{cccc}
\operatorname{Sim}_{11} & \operatorname{Sim}_{12} & \ldots & \operatorname{Sim}_{1 n} \\
\operatorname{Sim}_{21} & \operatorname{Sim}_{22} & \ldots & \operatorname{Sim}_{2 n} \\
\ldots & & & \ldots \\
\operatorname{Sim}_{m 1} & \operatorname{Sim}_{m 2} & \ldots & \operatorname{Sim}_{m n}
\end{array}\right]
$$


If one element in the matrix of semantic similarity $\operatorname{Sim}_{m n}=1$, corresponding element $c_{n}$ in original class vector of domain ontology $\operatorname{OWLClass}_{i n i}=\left\{c_{1}, c_{2}, \ldots, c_{n}\right\}$ will be chosen as a element of the final class vector and then a semantic space model will be established with the center of $c_{n}$.

The process of other four element vectors of domain ontology matching with the vector of knowledge module is similar with the class vector.

If the domain ontology is perfect enough, at least one element of five matrixes of semantic similarity is equal to one. In other words, at least one vector of the five elements final vectors of ontology is not empty. If all five vectors are empty set, it shows that the construction of domain ontology is not perfect or the whole framework is not reasonable or the knowledge modules don't belong to the domain.

When the element $\operatorname{Sim}_{m n}$ in matrix $\mathrm{S}$ is greater than a certain threshold $\operatorname{Sim}_{\text {threshold }}$, the key element $k_{m}$ in $K=\left\{k_{1}, k_{2}, \ldots, k_{m}\right\}$ is very similar to the element $c_{n}$ in $O W L C l a s s_{i n i}=\left\{c_{1}, c_{2}, \ldots, c_{n}\right\} \cdot k_{m}$ is very likely a concept in domain. So the $k_{m}$ is needed to be added in domain ontology.

The formula of Sim $_{\text {threshold }}$ is shown as (4):

$$
\operatorname{Sim}_{\text {threhold }}=\frac{\alpha}{m \times n} \sum_{i=1}^{n} \sum_{j=1}^{m} \operatorname{Sim}_{j i}+\beta \times \operatorname{Max}\left(\operatorname{Sim}_{j i}\right), \alpha+\beta=1
$$

The final vectors of domain ontology elements are formed after the vector of knowledge module matching with the five original vectors of ontology elements separately. The final vectors of domain ontology elements can be represented as following:

$$
\begin{aligned}
& \text { OWLClass }_{\text {fin }}=\left\{C_{1}, C_{2}, \ldots ., C_{i}\right\} \\
& \text { OWLIndividuals }_{f i n}=\left\{I_{1}, I_{2}, \ldots ., I_{j}\right\} \\
& \text { OWLObject Pr } \text { operty }_{\text {fin }}=\left\{O_{1}, O_{2}, \ldots, O_{k}\right\} \\
& \text { OWLData Pr operty } \text { fin }_{1}=\left\{D_{1}, D_{2}, \ldots ., D_{l}\right\} \\
& \text { OWLDescription }_{f i n}=\left\{\text { Des }_{1}, \text { Des }_{2}, \ldots ., \text { Des }_{m}\right\}
\end{aligned}
$$

OWLClass $_{f i n}$ represents the final vector of class. OWLIndividuals $s_{f i n}$ represents the final vector of individual. OWLObject $\operatorname{Pr}$ operty $_{\text {fin }}$ represents the final vector of object property. OWLData Property represents the final vector of data property. $O W L D_{\text {Description }}{ }_{f i n}$ represents the final vector of description.

\subsubsection{Semantic space generating}

At last, every element in the final vectors of domain ontology will be expanded according to the semantic relationship in domain ontology to form a semantic space model.

The steps to establish semantic space model centered on class are as following:

$\diamond$ Get all the object properties which are related with the class;

$\diamond$ Get the other related element through the object property. If the element is class, the expanding continue otherwise stop.

$\diamond \quad$ When the semantic distance between expanding class and the center class is greater than a certain stop value, the expanding will stop. The formula for semantic distance is shown as (5):

$\operatorname{dist}\left(o_{1}, o_{2}\right)=\left\{\begin{array}{c}\left(2^{-1-\text { level }(\text { comF })}-2^{-1-\text { level }\left(\text { como }_{1}\right)}\right)+\left(2^{-1-\text { level }(\text { comF })}-2^{-1-\text { level }\left(\text { como }_{2}\right)}\right) \\ 0\end{array}\right.$

comF is the nearest public parent class of $o_{1}$ and $o_{2}$ in ontology concept hierarchical tree. level $(\mathrm{comF})$ level $\left(\mathrm{o}_{1}\right)$ and level $\left(\mathrm{o}_{2}\right)$ represent the depth of node comF $o_{1}$ and $o_{2}$ in the ontology concept hierarchical tree.

The steps to establish semantic space model centered on individual, object property, data property are as same as the class. 


\section{Knowledge Requirement Analysis for the Design Activities}

In order to acquire the knowledge requirement swiftly and accurately and eliminate the semantic ambiguity, a knowledge requirement net is proposed in this paper. Knowledge requirement analysis is based on the relevant information of design activity. The information this paper considered includes the task of design activity, product object, the designer and the form of knowledge achievements.

1) Task of design activity: the task of design activity is a description of the task of current design activity, including the design requirements, performance index, completion time etc. The current task can be decomposed to several subtasks according to relevant requirement.

2) Product object: product object is a description of what object the current design activity is designing, that may be specific to product components.

3) Designer: designer is the person who is operating the current design activity. Due to different designer is different is the professional level and the ability to analyzing and solving problems, so designer should be considered in knowledge requirement analysis.

4) Form of knowledge achievements: what is the target of current design activity and what kind of knowledge will be generated in current design activity.

The process of knowledge requirement analysis is shown in Fig.3.

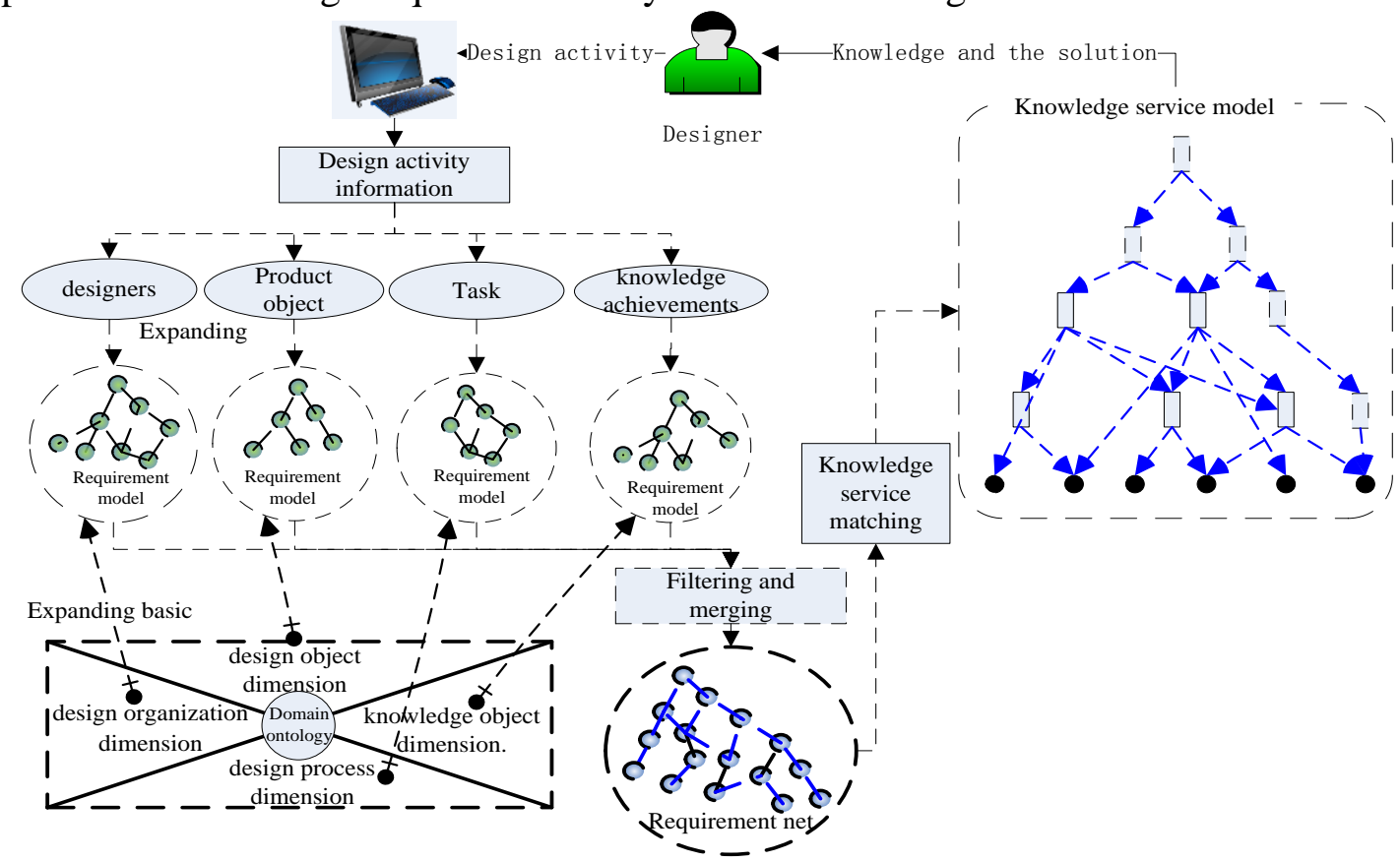

Fig. 3 The Process of Knowledge Requirement Analysis

First of all, the knowledge requirement models will be constructed according to the basic information of design activity that including product object requirement model, designer requirement model, activities task requirement model and knowledge achievements requirement model.

The four knowledge requirement models are constructed based on the corresponding dimensions of domain ontology. Eventually four knowledge requirement models through certain filtering and merging rules formed a complete knowledge requirement net.

\subsection{Knowledge Requirement Model.}

Knowledge requirement model is formed through domain ontology expansion based on the basic information of the design activities. The same information of the design activities may be described differently, so in the process of requirement model generating how to eliminate the semantic ambiguity between concepts and relationship conflicts is needed to consider. The knowledge requirement model generating follows the next steps:

$\diamond$ Segment the describing sentences of the basic information of the design activities and extract important concepts in domain.

$\diamond$ Match the concepts with the classes in ontology. Successful matching classes will expand based on semantic relationship in ontology to form requirement model; 
$\diamond$ Convert the knowledge requirement model to OWL format for storage.

\subsection{Knowledge Requirement Net.}

Knowledge requirement net is a merging model of requirement models that could represent the knowledge requirement of design activities completely.

Definition 3: Knowledge Requirement Network can be defined as a five-element model

$$
\mathrm{KRN}=\{\mathrm{PO}, \mathrm{DP}, \mathrm{DM}, \mathrm{KA}, \mathrm{MA}\}
$$

PO represents the product object model. DP represents the designer model. MD represents the design task model. KR represents the knowledge achievements model. MA represents the merging algorithm. The merging technology of requirement model is oriented to OWL format. The process of merging is shown as Fig.4.

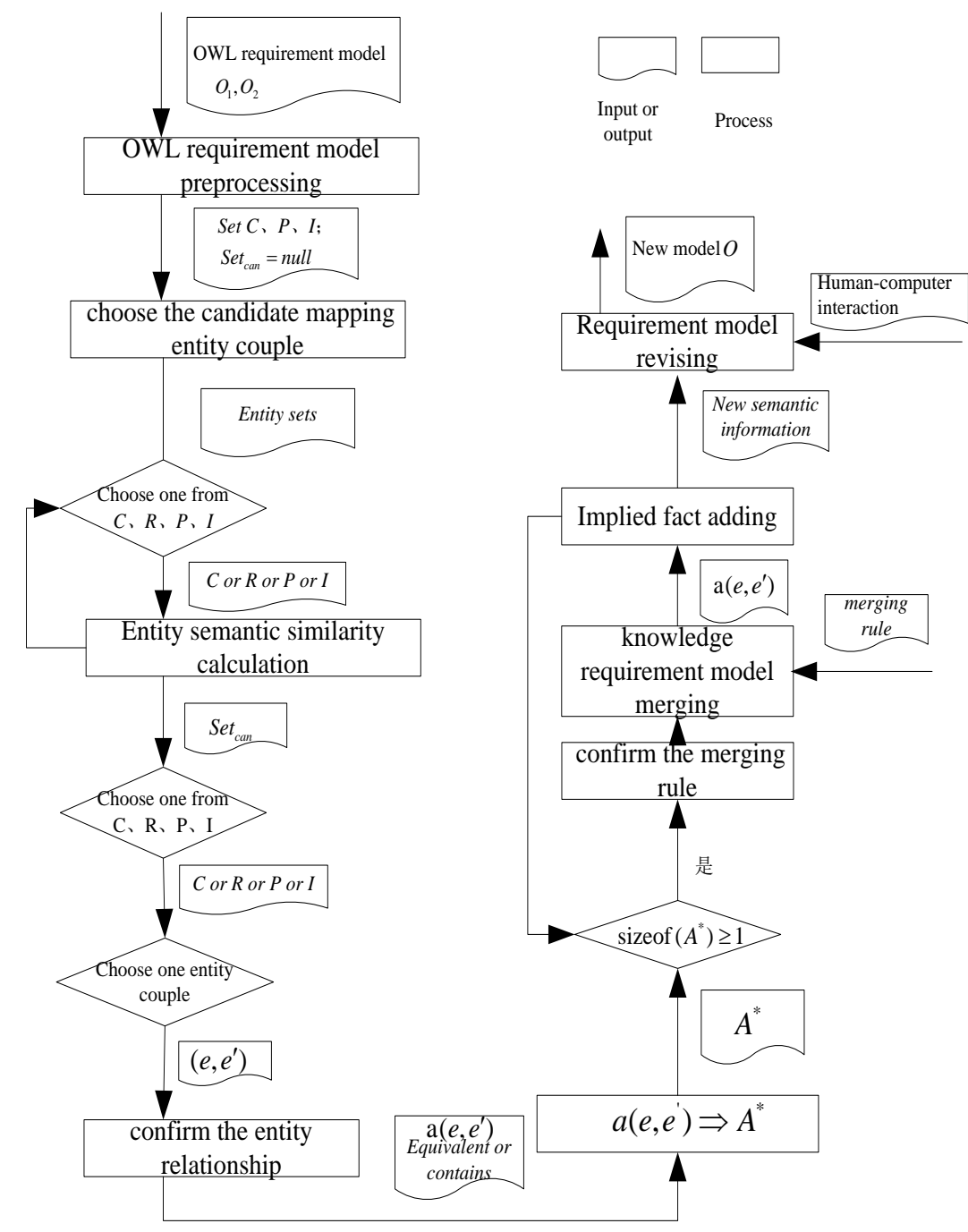

Fig. 4 The Process of Requirement Model Merging

\section{Knowledge Service Matching}

Knowledge service matching is a process to get the exact knowledge through knowledge requirement net matching with the knowledge service model. The matching process is based on semantic unit.

Definition4: semantic unit: semantic unit is a partial semantic structure of any element $C$ in knowledge requirement net or the semantic space model and the set of its all related elements $\left\{C_{1}, C_{2}, C_{3} \ldots C_{n}\right\}$ and the set of all related semantic relationship $\left\{R_{1}, R_{2}, R_{3} \ldots R_{n}\right\} \cdot$

Knowledge requirement net matching with the knowledge service model follows the next steps: 1) cut knowledge requirement net to semantic unit; 2) cut semantic space to semantic unit; 3) semantic 
similarity calculation between semantic units; 4) get related knowledge module according to the matching result.

\subsection{Semantic Units Generating.}

Because the knowledge requirement net and semantic space are eventually stored in OWL format, so ontology parsing technology is used to generate semantic unit. Firstly, the sets of independent elements are formed that include class set, individual set, object property set, data property set. And then the related elements will be got centered on the elements in the set until all the elements in the sets already formed semantic units. In theory, there are as many elements in semantic space as there are semantic units. The process of semantic units generating is as shown in Fig. 5.

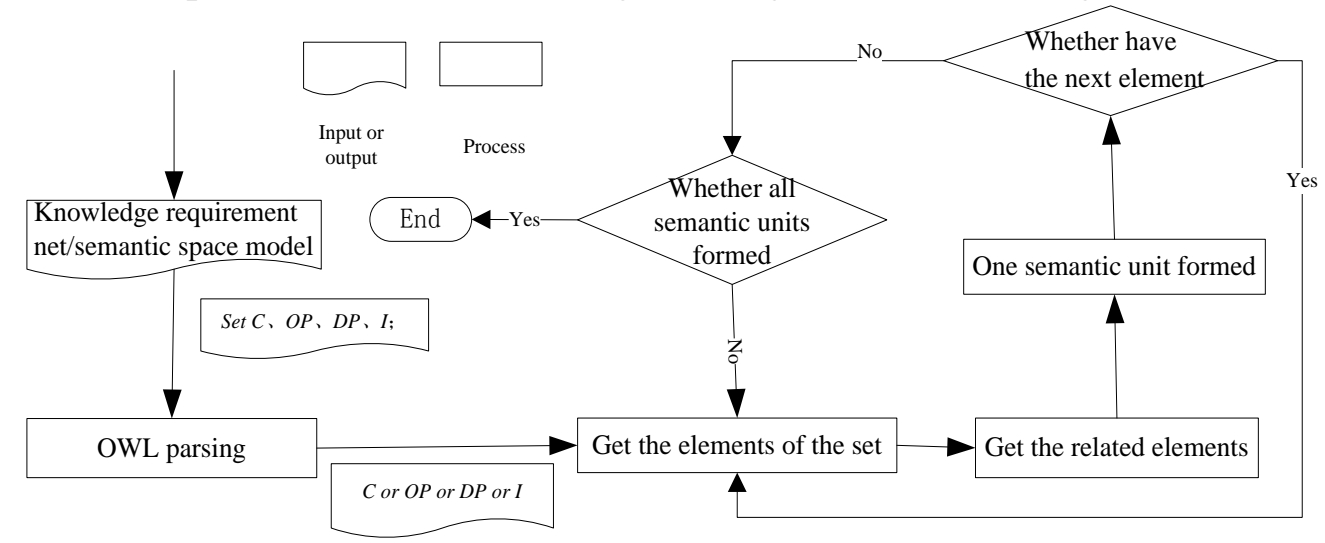

Fig. 5 Semantic Units Generating

\subsection{Similarity Calculation between Semantic Units.}

Definition5 similarity between semantic units: The semantic similarity between semantic units Q and D is a sum of the semantic similarity between all node in $\mathrm{Q}$ and all node in D.

The calculation of semantic similarity between two semantic units is essentially calculation of similarity between two elements in semantic units. The semantic distance is calculated firstly according to (6):

$$
\begin{aligned}
\operatorname{Ditance}\left(e_{1}, e_{2}\right) & =\left(I C\left(e_{1}\right)-I C\left(n c a\left(e_{1}, e_{2}\right)\right)+I C\left(e_{2}\right)-I C\left(n c a\left(e_{1}, e_{2}\right)\right)\right) \\
& =I C\left(e_{1}\right)+I C\left(e_{2}\right)-2 \times I C\left(n c a\left(e_{1}, e_{2}\right)\right) \\
& =-\log P\left(e_{1}\right)-\log P\left(e_{2}\right)+2 \times \log P\left(n c a\left(e_{1}, e_{2}\right)\right)
\end{aligned}
$$

$\operatorname{Ditan} c e\left(e_{1}, e_{2}\right)$ is the semantic distance between two elements. $P\left(e_{1}\right)$ is the appearing frequency of element $e_{1}$ in the domain ontology. $P\left(e_{2}\right)$ is the appearing frequency of element $e_{2}$ in the domain ontology. $P\left(n c a\left(e_{1}, e_{2}\right)\right)$ is the appearing frequency of the nearest common parent node of the two elements in domain ontology.

Then the semantic similarity can be calculated according to (7):

$$
\operatorname{Sim}\left(e_{1}, e_{2}\right)=\frac{1}{\operatorname{Ditan} c e\left(e_{1}, e_{2}\right)}
$$

Finally, the semantic similarity between two semantic units can be calculated by adding all the semantic similarity between two elements in semantic units.

When the semantic similarity between two semantic units is greater than a certain threshold, the corresponding semantic space model is the needed one, and then the needed knowledge will be got according to the semantic space model. At last, the result of knowledge service return to the user in a format of knowledge solution tree.

\section{Case Study}

This study is validated in a military-vehicle design knowledge service prototype system. The domain ontology is established with protege4.1. Then the knowledge requirement net and the knowledge service model are formed based on the domain ontology. At last, the knowledge service return to the user in a format of knowledge solution tree through the service matching algorithm. The 
design-knowledge services can be provided accurately and swiftly to the users by the military-vehicle design knowledge service system. For example, a designer wants to make an overall program for the vehicle design, and this requirement is descripted in the activity. Then the former solutions and the related resources will be return in the format of knowledge solution tree.

Based on the proposed techniques for knowledge service, a prototype of knowledge service mechanism for product design process was implemented. Computer hardware in the implementation environment is equipped with an application server, a web server, and a data and knowledge server. The application programs are implemented using JAVA language as the development tool, Oracle 10g version for database. There is already a knowledge management system in the Laboratory, and the knowledge service mechanism is added into the original system as a new module. The knowledge management system for product design process mainly includes five modules and their relationship is shown in Fig.6.

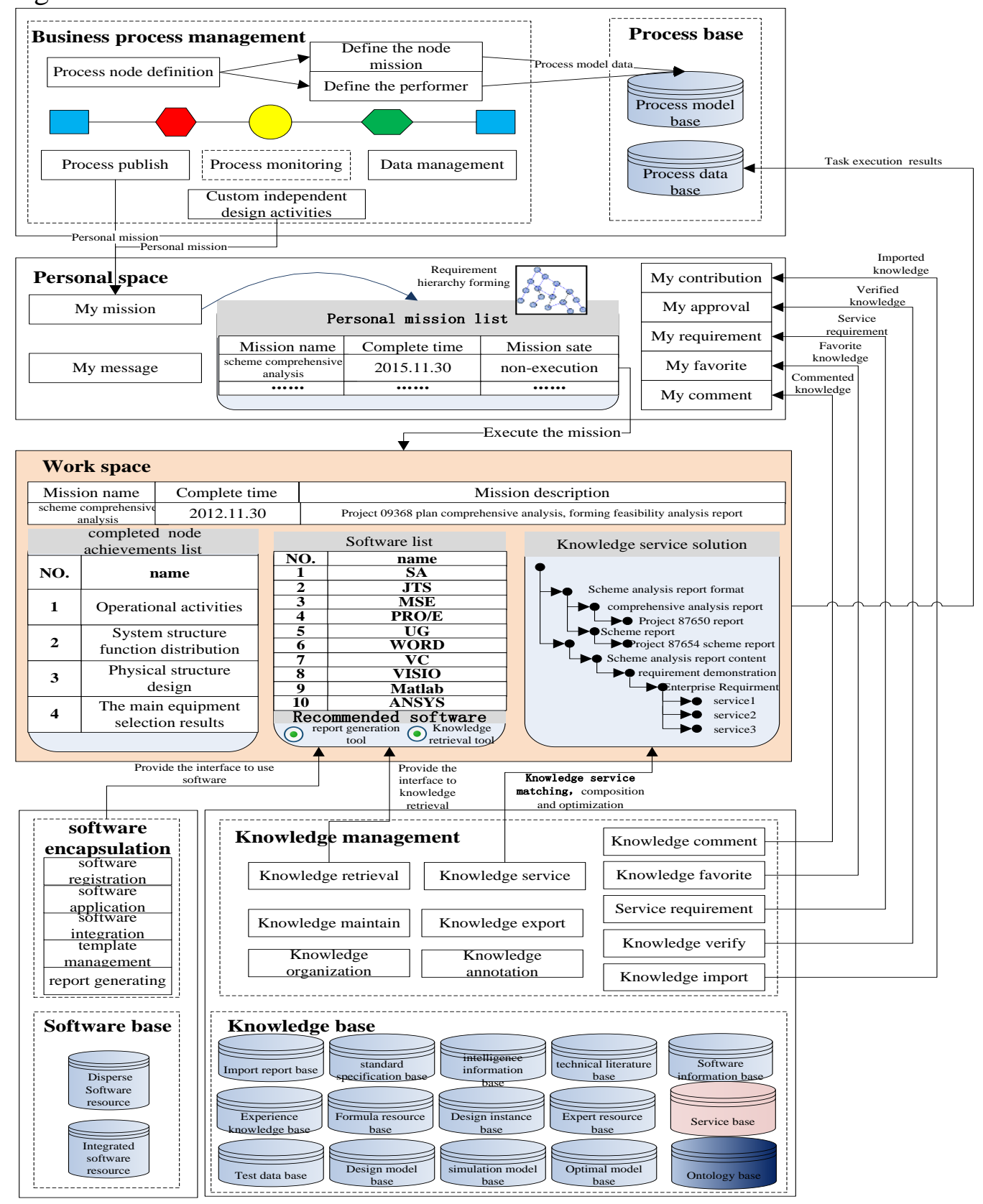

Fig. 6 The Knowledge Management System Business Relations

Firstly, a product design process should be defined in the business process management module, in the meanwhile, the design tasks and performers are defined. When the process is published, the performers will receive their own task in the personal space. The performers can choose one task to do and the corresponding work space emerges. In the work space, there are three aspects information: 1) 
the results list of executed process node; 2) the software list which is may needed to perform this task, at the same time, the recommended software will be given automatically; 3)the knowledge service solution.

The performers in work space can conveniently reference the results produced by the other process node and the result of the knowledge service, and the soft-wares are also convenient to use. When the task completed, the related data and results will be stored in the process database, and the data version history of each process node is also stored.

Software encapsulation module is used to provide interface to call the related software and knowledge management module provide knowledge retrieval and knowledge service interface. On the other hand, knowledge management and service module can work as an independent module. Users in the knowledge management module can some independent operations, such as favorites, commenting, and importing and so on, and the corresponding operations will be recorded in the personal space.

The system function diagram is shown in Fig.7. The knowledge service module not only provides the knowledge service solution for the work space but also can be used as an independent module.

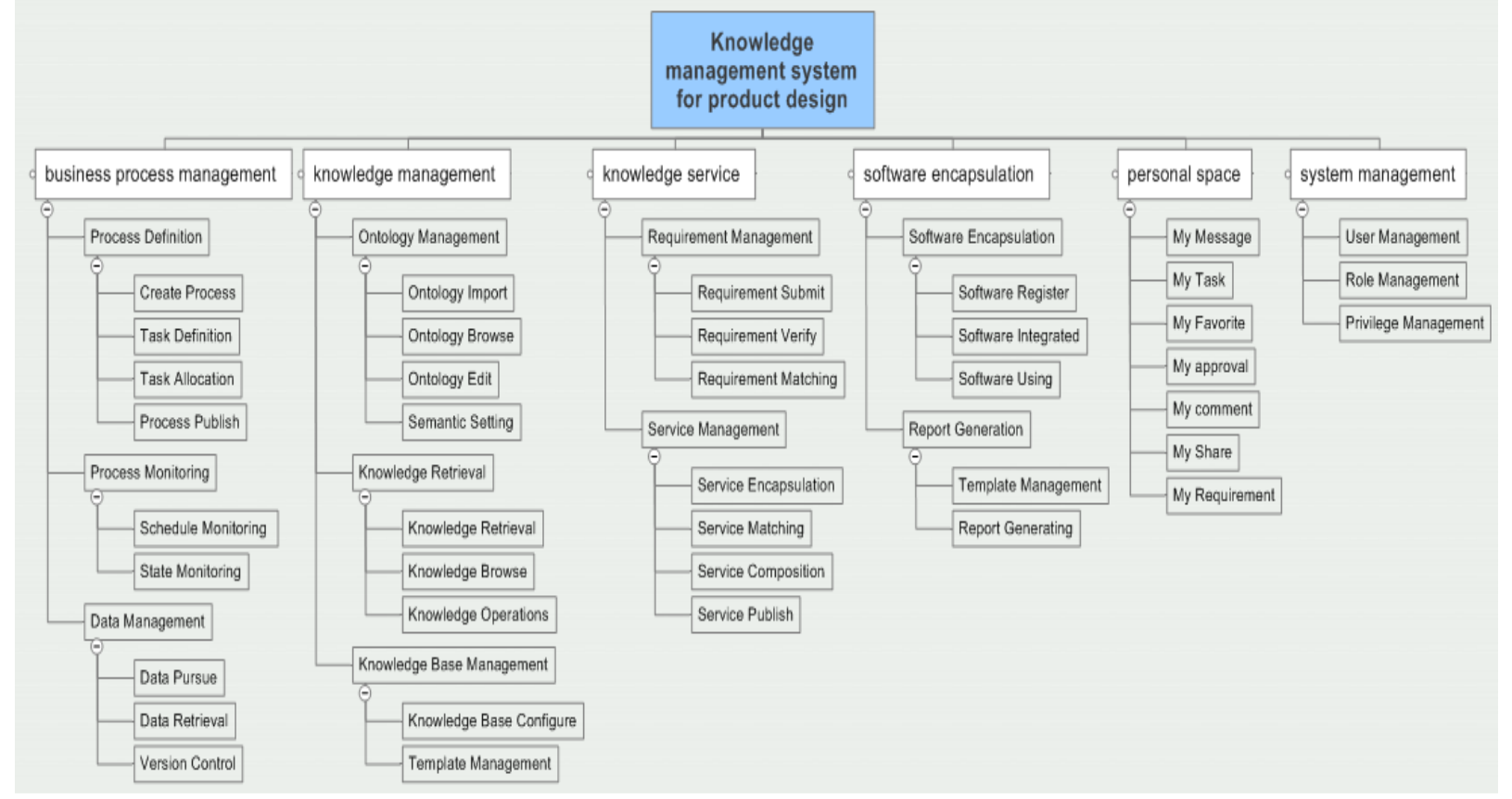

Fig. 7 the System Function Diagram

When user have a knowledge service requirement, the requirement should be submitted in the "My Requirement" module. If the requirement meet the standard conditions, the requirement is formed a requirement hierarchy, and then the semantic spaces on the node of requirement hierarchy match with the semantic spaces on the knowledge services. The candidate service list will be found after the similarity calculation and the final knowledge service solution will be formed after service composition and optimizing.

Fig.8 presents the user interface for the knowledge service requirement submitting, while Fig.9 shows the knowledge service solutions. Service solutions are in form of requirement hierarchy, and each node associate with a knowledge service. As Fig.9 shows, the road wheel design service contains the knowledge about "road wheel selection criteria", "Pmm calculation and reference data","road wheel reference data" and so on. 


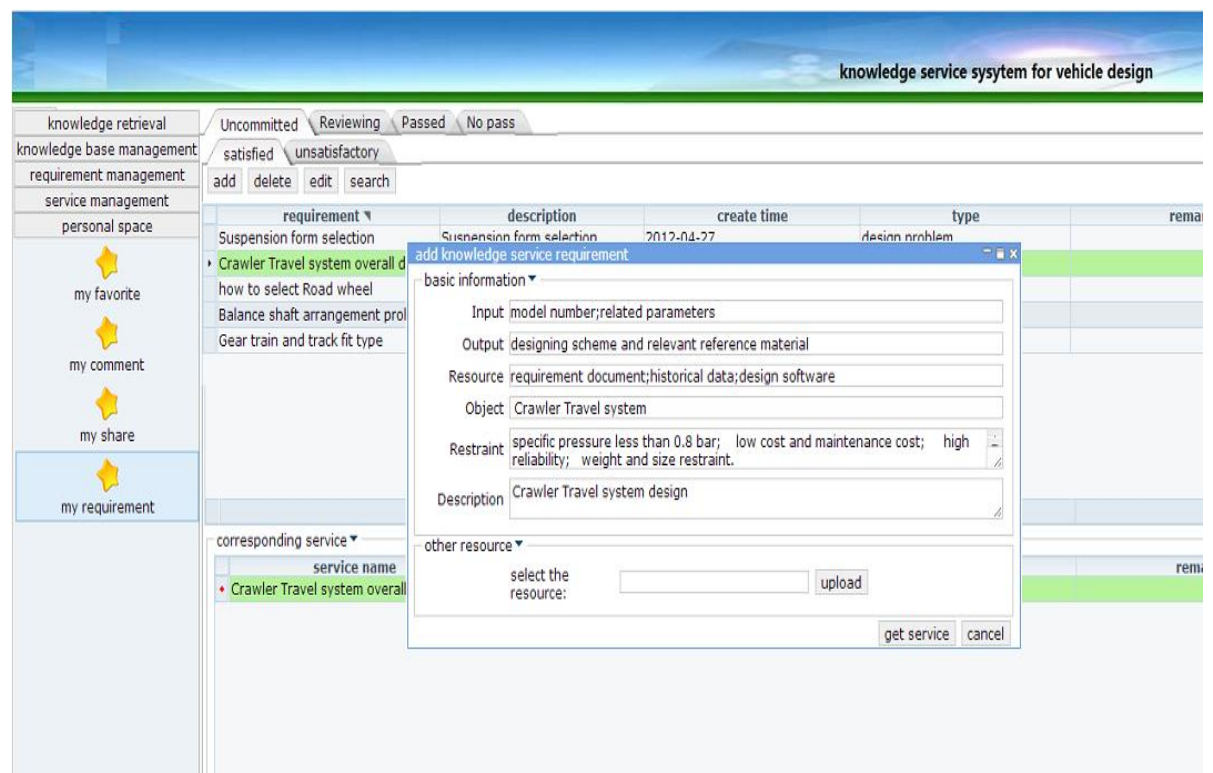

Fig. 8 Knowledge Service Requirement Submitting

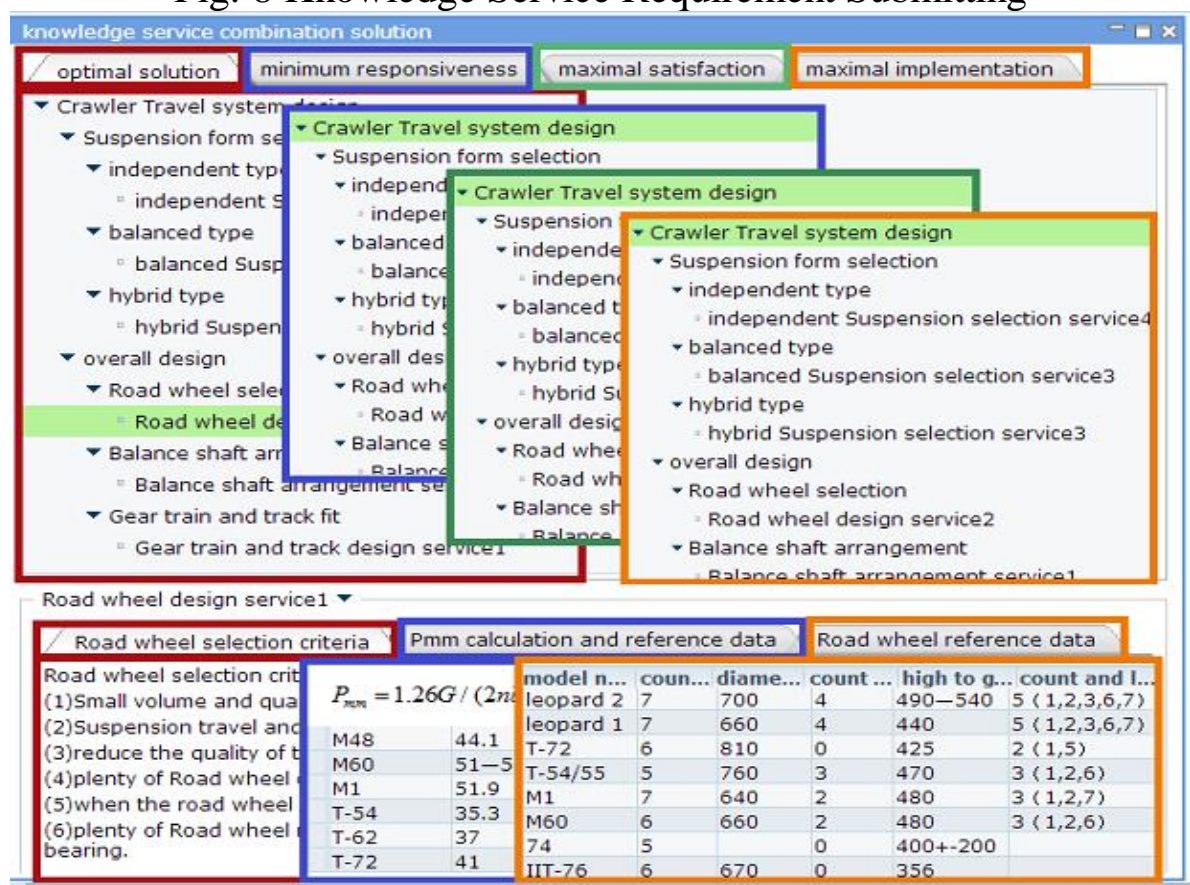

Fig.9 Knowledge Service Solutions

Knowledge service provides a full solution for one problem to the user and make the knowledge have a strongly logic structure. Since the service matching process is based on semantic, compared to the traditional knowledge retrieval and service matching approach, our method in this paper is more efficient and can get the knowledge which is the user really needed.

This service system have been tried and tested in a military vehicle design department, and the satisfaction of this system is tested through the way of questionnaire survey. The survey result shows that more than $90 \%$ of the respondents are satisfy with the knowledge service mechanism and more than $98 \%$ of the respondents think that this service mechanism is superior to the traditional knowledge retrieval approach.

\section{Summary}

This paper proposed a framework for knowledge service oriented to design activities. Designers can rapidly and seamlessly access entire product life-cycle knowledge with the framework. The knowledge representation method and the knowledge service model based on semantic space are proposed in this paper. They are the foundation of knowledge service. And then the method of 
knowledge service matching is given that make the efficiency and the accuracy of knowledge service greatly increased. The result of case study proved that the developed framework and its architecture and involved methods are applicable for effective knowledge service in practice.

Further works include: to develop entire domain ontology modeling, to integrate more methods which is suitable for designers, such as the roadmap, to propose a method that make the knowledge rise in value.

\section{Acknowledgements}

This work is supported by innovation Fund of China Electronics Technology Group Corporation, (Project Number: E201502002). We are grateful to our partner company for their support of our research.

\section{References}

[1]. Chen Si,Yan Yan,Wang Guoxin, et al, "Product-design knowledge retrieval based on ontology," Journal of Beijing Institute of Technology,vol.20(2012),no.3,p. 79-386.

[2]. YANG Tao, WANG Yun-li, XIAO Tian-yuan, et al, "Research on Personalized Active Design Knowledge Service Systems," Computer Integrated Manufacturing Systems, vol.8 (2002), no.12, p. 950-953, 959.

[3]. WANG Jun, PAN Xing, and LIU Lu, "Research on agile knowledge reuse based on multi-Agent \& knowledge service," Computer Integrated Manufacturing Systems, vol.12 (2006), no.6, p. 840-846.

[4]. Gregoris M, Kostas K, and Panos G, "Knowledge services on the semantic web," Communications of the ACM, vol.50 (2007), no.10, p. 53-58.

[5]. Bhatta M, Rahayu W, and Soni S P, "Ontology driven semantic profiling and retrieval in medical information systems," Web Semantics: Science, Services and Agents on the World Wide Web, vol.7(2009), no.4, p. 317-331.

[6]. Lim S C J, Liu Y, and Lee W B, "Multi-facet product information search and retrieval using semantically annotated product family ontology," Information Processing and Management, vol.46 (2010), no.4, p. 479-493.

[7]. DIAO Xing-chun, TAN Ming-chao, and CAO Jian-jun, "New method of character string similarity compute based on fusing multiple edit distances," Application Research of Computers, vol.27 (2010), no.12, p. 4523-4525.

[8]. Yu Haiying, "The Comparison of the LCS Algorithm with the GST Algorithm in Strings Similarity Metrics," Electronic Science and Technology, vol.24 (2011), no.3, p. 101-103,124.

[9]. S. C.Johnson, Y.Liu, W BLee.A methodology for building a semantically annotated multi-faceted ontology for product family modeling[J].Advanced Engineering Informatics, vol.25(2011), no.2, p. 147-161.

[10]. F.Q. Song, G. Zacharewicz, D. Chen.An ontology-driven framework towards building enterprise semantic information layer[J].Advanced Engineering Informatics, vol.27(2013), no.1, p. 38-50.

[11]. R.R. Starr, J. M. P.Oliveira. Concept maps as the first step in an ontology construction method [J].Information Systems, vol.38 (2013), no.5, and p.771-783.

[12]. LiuY, Zheng XL,Tang F.Ontology design with a granular approach[J]. Expert Systems with Applications, vol.41 (2014), no.10, p. 4867-4877.

[13]. Asanee Kawtrakul. Ontology Engineering and Knowledge Services for Agriculture Domain [J]. Journal of Integrative Agriculture, vol.11 (2012), no.5, p. 741-751. 